\title{
Optimum Design of a Flexible Wing Structure to Enhance Roll Maneuver in Supersonic Flow
}

\author{
Duane E. Veley, Narendra S. Khot, Jeffrey V. Zweber
}

Structures Division, Air Vehicles Directorate, Air Force Research Laboratory

\author{
Alicia R. Hartong \\ Wright State University
}

\section{Abstract}

This work combines two efforts that have been on-going in the Air Force Research Laboratory. The first effort is the work of the Design Modeling Group which is using the Adaptive Modeling Language (AML) to develop an objectbased technology assessment system. One of the advantages of this effort is that the analytical tools (legacy codes) that are used may be integrated into the AML environment one at a time, thereby providing many intermediate products each of which may enhance productivity. So while this effort is still in its infancy, a graphical user interface to the Automated STRuctural Optimization System (ASTROS) has been developed and is used in this effort to develop a parametrically defined finite element model. The second effort is the work on designing flexible wings without ailerons for performing roll maneuvers. Here a flexible wing is optimized for weight with constraints on strength for a 9-g symmetric pull-up maneuver at $\mathrm{M}=0.85$ at $30 \mathrm{psi}$ and on the modal frequency distribution. Differential wing twist and camber is achieved by providing a system of actuating elements distributed within the internal substructure of the wing to provide roll control power. The modal approach was used to develop equilibrium equations for the steady roll maneuver of a wing subjected to aerodynamic loads and actuating forces. The distribution of actuating forces to achieve a specified flexible roll rate was determined by using an optimal control design approach. Here, full-scale wings are considered for the assessment of strain energy as a measure of the necessary power required to produce the antisymmetric twist and camber deformation to achieve the required roll performance. The wings used here break the paradigm of the low aspect ratio wings typical for supersonic vehicles. The purpose of this is to enhance the flexibility of the wing and to reduce the control power needed to twist the wing.

\section{Introduction}

The purpose of the technology assessment system ${ }^{1}$ that is being developed in the Adaptive Modeling Language (AML) is to look at how the new technologies and concepts affect aircraft design and cost. New technologies can be assessed as enhancements to existing air vehicles or as concepts for new vehicles. Several areas of the technology assessment system that are currently under development include a new paradigm for conceptual design tools, ${ }^{2}$ conceptual design tools for hypersonic vehicles, a graphical user interface ${ }^{3}$ for the Automated STRuctural Optimization System (ASTROS), ${ }^{4}$ automatic differentiation ${ }^{5}$ and optimization. ${ }^{6}$ This work uses the graphical user interface for ASTROS to develop an initial finite element model for ASTROS and a second finite element model based on the first model with some minor planform changes. Using the parametric relations defined for the first model, a second model is generated rather quickly and with minimal effort.

Each of the models generated for ASTROS are optimized for minimum weight and the distribution of actuating forces to achieve a specified flexible roll rate is determined. The purpose of this portion of the effort is to assess the potential of recovering some of the roll effectiveness that is lost due to the flexibility of the wing (see Fig. 1) by using actuators to control the deformation of the wing. ${ }^{7}$ ASTROS uses the Unified Subsonic and Supersonic Aerodynamic Analysis (USSAERO) ${ }^{8}$ program to compute the aerodynamic loads on the aircraft wing. This approach uses a superposition of vortex singularities applied to a discrete number of aerodynamic panels to calculate the discrete pressure distribution over the wing surface.

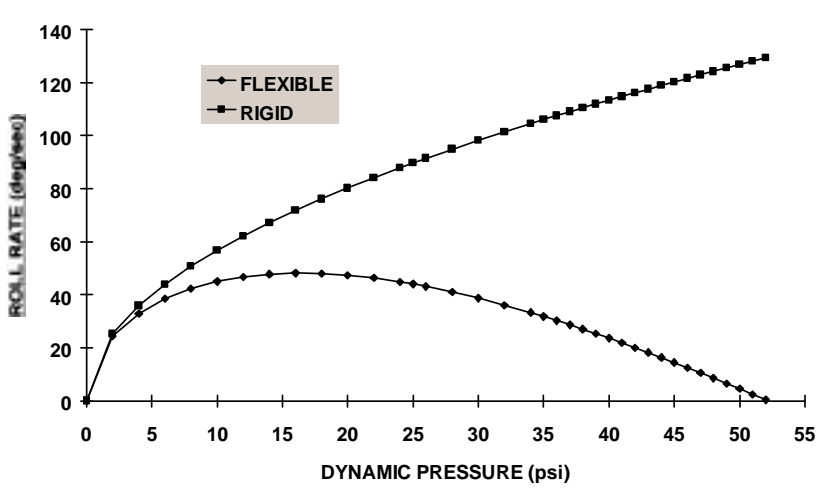

Figure 1. Effect of flexibility on the roll rate of a typical wing for various dynamic pressures. 
The traditional method of using ailerons to produce a rolling moment is effective at low dynamic pressures where the wing is essentially "rigid." However, at high dynamic pressures, where the deformation of the wing is significant, the roll rate is reduced by a detrimental aerodynamic twisting moment resulting from the trailing edge control surface rotation.

\section{The Adaptive Modeling Language and the Graphical User Interface to ASTROS}

A brief description of the Adaptive Modeling Language (AML) is in order to help understand some of the benefits of the Graphical User Interface (GUI) that was developed for ASTROS. AML is an object-oriented, knowledge-based, programming language developed by TechnoSoft, Inc. AML has several unique capabilities which make it an excellent language for designing engineering systems. These capabilities include dependency tracking, demand-driven calculations (lazy evaluations), adaptive class structure, and the flexibility of properties to be of any class. A more thorough description of these capabilities is given by Veley. ${ }^{6}$

The GUI that was developed for ASTROS is more than just a geometry sketcher. Properties other than geometry are defined in the GUI such as material properties and loading conditions as well as properties such as design variables and design constraints that are used to define an optimization problem. Using a single geometric definition and the nongeometric information, both a finite element model and an aerodynamic model are generated.

At the current stage of development, the GUI does not define a complete ASTROS data deck. The bulk data that is missing for this problem are those entries that are unique to the modified version of ASTROS that is being used to handle the control system design. Also, a comprehensive generic solution control object which the user can tailor to suit the user's model is still under development.

Dependency tracking and demand driven calculations permit parametric relations to be updated automatically. If a change is made to the sweep angle, span or chord length, the ribs and spars will conform to the new planform. Similarly, a change in the airfoil shape will cause the height of the ribs and spars to change accordingly.

The adaptive class structure of AML allows for ease in redefining a model. Ribs, spars and actuators may be added and deleted from the model with a few clicks of the mouse button. These features provide much ease in creating new models from existing models and significantly reduces the time to develop additional models.

\section{The Flexible Wing}

The state-of-the-art in design optimization software falls somewhat short of the need to simultaneously consider all the design requirements from the various disciplines connected with aircraft design. Thus, obtaining an "optimum" design in an integrated fashion is not yet possible. This leads to the design process consisting of a combination of optimizations based on important design requirements and then using this "optimized" design as a basis for modifications to satisfy other design or performance requirements from other disciplines. This multiple step design approach may be necessary to satisfy global and local requirements form different disciplines.

The approach used in this effort is a two step process. First, an optimum design satisfying strength and frequency distribution requirements is obtained. This optimization problem is solved using ASTROS version 20 for a design condition of 9-g symmetric pull-up maneuver at $\mathrm{M}=0.85$ at 30 psi. Second, using a modified version of ASTROS, the control system is generated to twist and camber the optimum wing to achieve the target flexible roll rate.

One of the main elements of the flexible wing concept is that rolling moment control is achieved by controling the wing twist and camber. The elastic wing twist and camber needed for control are achieved through linear actuators mounted diagonally across the internal structure. These actuators are modeled as rods exerting tensile/compressive loads to twist and camber the wing to achieve specified roll rates. This permits assessments between energy requirements for twist and camber control to achieve the required roll performance at various dynamic pressures.

The equilibrium equations for steady roll maneuver can be written as ${ }^{7}$

$$
[K]\{x\}+q[T]^{T}[A]\{\alpha\}+[F]\{u\}=0
$$

where $[K]$ is the structural stiffness matrix of the finite element model, $\{\mathrm{x}\}$ is the vector of nodal displacement vector, $\mathrm{q}$ is the dynamic pressure, $[\mathrm{T}]$ is the transformation matrix from structural degrees of freedom to the aerodynamic degrees of freedom, $\{\alpha\}$ is the vector of angle of attack at the aerodynamic panels, $[F]$ is the applied actuator load distribution matrix and $\{u\}$ is the vector of actuator stimuli. The product $[F]\{u\}$ is the vector of control forces generated at the structural node points due to the actuator forces $\{u\}$ or due to the voltages applied to the solid state actuators.In the latter case the elements of matrix $[F]$ would depend on the number of stacks, number of cycles and the properties of the solid state actuators in addition to the direction cosines associated with the actuators.

The displacement vector $\{x\}$ can be defined as a linear combination of rigid body modes and vibration modes as 


$$
\{x\}=\left[\Psi_{r}\right]\left\{\eta_{r}\right\}+\left[\Psi_{e}\right]\left\{\eta_{e}\right\}=[\psi]\{\eta\}
$$

where $\left[\Psi_{r}\right]$ is the rigid body mode, $\left[\psi_{e}\right]$ is the specified number of antisymmetric low frequency modes, $\left\{\eta_{r}\right\}$ is the generalized rigid body displacements and in present case it is equal to the roll angle $\phi .\left\{\eta_{e}\right\}$ in Equation (2) is the elastic displacements. The subscript $\mathrm{r}$ and e are used to indicate rigid body and elastic vibration modes.

The angle of attack $\{\alpha\}$ at the control points of aerodynamic panels can be written as

$$
\{\alpha\}=\frac{1}{V}[T]\{\dot{x}\}
$$

where $V$ is the free stream velocity. Using Equation (2) the angle of attack can be written as

$$
\{\alpha\}=\frac{1}{V}[T]\left[\left[\Psi_{r}\right] p+U\left[\frac{\partial \psi_{e}}{\partial \bar{x}}\right]\left\{\eta_{e}\right\}\right]
$$

where $\mathrm{p}$ is the roll rate $\dot{\phi}, U$ is the chord wise component of $V$ in the direction $\bar{x},\left[\frac{\partial \psi_{e}}{\partial \bar{x}}\right]$ is the matrix of flexible mode gradient with respect to the $\bar{x}$ coordinate. Equation (4) can be written as

$$
[\alpha]=\left[\frac{\bar{\Psi}_{r}}{V}+\frac{\partial \bar{\Psi}_{e}}{\partial \eta_{e}}\right]\left\{\frac{p}{\eta_{e}}\right\}
$$

where $\bar{\Psi}_{r}$ and $\bar{\Psi}_{e}$ are the rigid and vibration modes expressed in aerodynamic degrees of freedom. Using Equation (5) the equilibrium Equation (1) can be written in terms of generalized coordinates as

$$
\begin{gathered}
{[\bar{K}]\left\{\frac{p}{\eta_{e}}\right\}+q[\bar{A}]\left\{\frac{p}{\eta_{e}}\right\}+[\bar{F}][u]=0} \\
\text { where }[\bar{K}]=\left[\begin{array}{cc}
0 & 0 \\
0[\Psi]^{T}[K][\Psi]
\end{array}\right] \\
{[\bar{A}]=[\Psi]^{T}[T]^{T}[A]} \\
{[\bar{F}]=[\Psi]^{T}[F]}
\end{gathered}
$$

Solving Equation (6) for the generalized coordinates gives

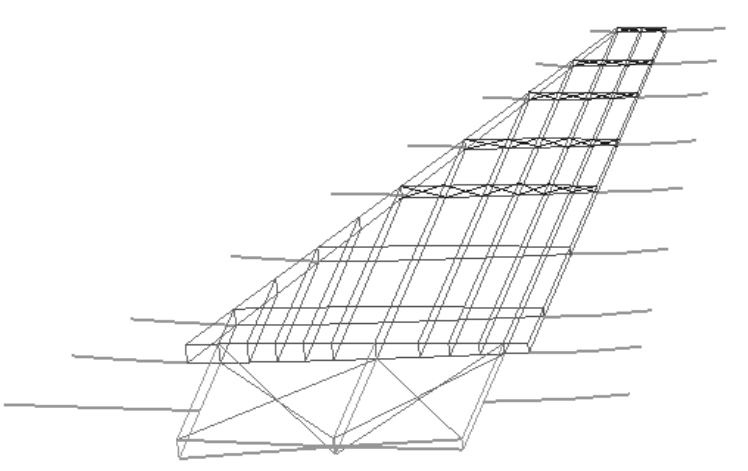

Figure 2. Finite element model of first wing

$$
\begin{gathered}
\left\{\frac{p}{\eta_{e}}\right\}=[[\bar{K}]+q[\bar{A}]]^{-1}[\bar{F}]\{u\}, \\
\text { or }\left\{\frac{p}{\eta_{e}}\right\}=\left[\begin{array}{l}
B \\
C
\end{array}\right]\{u\}
\end{gathered}
$$

The roll rate $p$ is given by

$$
p=[B]\{u\}
$$

and the generalized coordinates of the elastic modes are give by

$$
\left\{\eta_{e}\right\}=[C]\{u\}
$$

For the specified distribution of actuator forces $\{u\}$ Equation (13) can be used to calculate the roll rate $p$. However, for

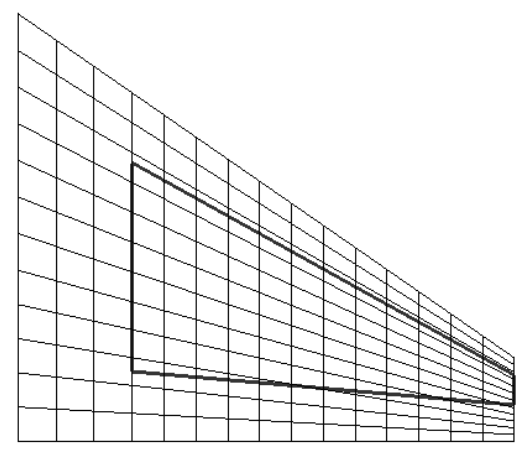
achieving the target roll rate $p_{t}$ at specified Mach number and specified dynamic pressure, the optimal control design approach was used to determine the distribution of actuator forces necessary to deform the wing. 


\section{Application}

The Adaptive Modeling Language (AML) Graphical User Interface (GUI) developed for ASTROS was used to model two similar generic fighter wings. The finite element model developed for the first wing which is more typical of fighter wings is shown in Fig. 2 and the aerodynamic model of the same wing is shown in Fig. 3. The semi-span (fuselage to tip) of the first wing is 180 in and the root chord is $163.9 \mathrm{in}$. In order to demonstrate the effect of flexibility in the wing, the second wing (shown in Fig. 4) is a modified version of the first wing in which the semi-span and root-chord were changed to be 246 in and 128.75 in, respectively. These values were chosen to maintain the same wing area for both wings.

In order to assess the performance of the AML-GUI as a model generator and modifier, a timing summary was kept of each of the activities that constitute model development. The timing summary is given in Table 1 .

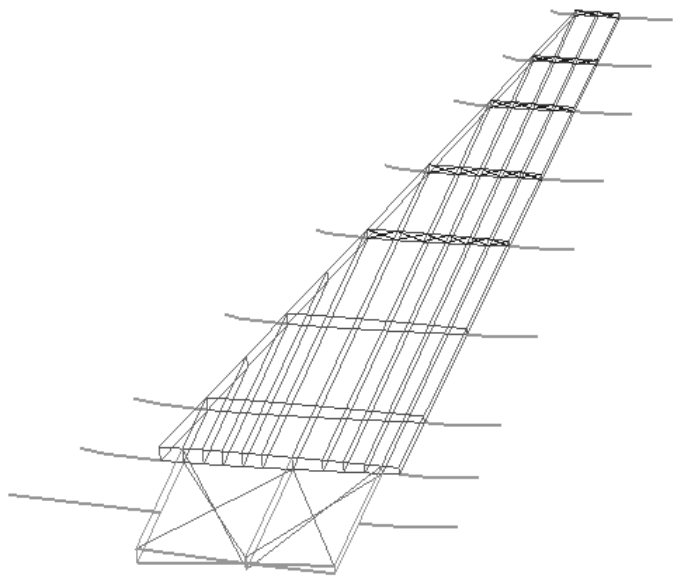

Figure 4. Finite element model of modified wing

Not included in the summary is the time spent reviewing and checking the entered data. The total time in front of the computer for defining the first wing was approximately 2 hours and for the second wing approximately one half hour. These times represent the time of someone who is familiar with the program and has all of the data ready to enter into the program. Note that the amount of data needed to be computed ahead of time is also reduced in that much of the data is "translated" from one form to another within the program.

The functions timed are as follows: The definition of the planform which includes the span, root chord, tip chord, airfoil shape, twist camber, etc. The substructure step includes laying out the drafting lines that help in the definition of the substructure, and the placement of the wing box, ribs, spars, actuators and the wing attachment. The properties step is assigning the initial sizes and the materials (which come from a material catalogue that is used in several AML programs) to the wing components. The extra points and boundary conditions (BCs) include the definition of additional "drafting" points, RBARs, MPCs, SPCs and SUPORT conditions. The time to generate the extra points is approximately the same as the time to generate the boundary conditions. The masses step is the addition of non-structural masses to the model. The aero grid step is the definition of the divisions of the planform used to generate the aerodynamic grid. The FE mesh step includes the time to generate a finite element mesh, define design constraints, define the eigenvalue

Table 1: Timing Summary of Model Creation (min:sec)

\begin{tabular}{|c|c|c|}
\hline Step & Wing 1 & Wing 2 \\
\hline \hline Planform & $0: 42$ & $0: 12$ \\
\hline Substructure & $14: 23$ & - \\
\hline Properties & $3: 33$ & - \\
\hline Extra Points/BCs & $24: 50$ & $9: 57$ \\
\hline Masses & $2: 30$ & - \\
\hline Aero Grid & $1: 07$ & - \\
\hline FE Mesh & $10: 00$ & $10: 00$ \\
\hline \hline TOTAL & $58: 00$ & $20: 10$ \\
\hline
\end{tabular}
solution method and write out the ASTROS data deck.

The finite element mesh is completely regenerated each time and is for the most part a push the button and get a cup of coffee type function, whereas the rest of the development time is nearly constantly hands on inputting of data.

It is worthy of note from the development time summary that the primary change in the model occurs at the planform step (a conceptual level definition) and that change would generally affect everything that follows. However, with the dependent relationships between each function, as the span and the root chord change, the substructure, masses and aerodynamic grid move with it and do not need to be redefined. The initial properties for the optimization are the same and, thus do not need to be changed. Unfortunately, the current method used to define the extra points is not linked to the geometry and so those points do not move with the geometry as the wing is stretched. Consequently, the extra points and associated boundary conditions need to be redefined. However, those boundary conditions that are not associated with the extra points that were relocated do not need to be redefined. Although the parameters for defining the finite element mesh are dependent on other properties, the mesh itself is not and needs to be regenerated each time. While this may be somewhat time consuming for large meshes, it is not labor intensive so the engineer may perform other tasks while the finite element mesh is being computed. Although no relationship changes were made to the substructure, properties, masses or aero grid in this example, the capability does exist. 
The two wings were initially designed for a 9-g symmetric pull-up maneuver at Mach 0.85, and 30 psi. The optimized designs were then sent to a modified version of ASTROS with anti-symmetric boundary conditions to generate data required for designing the control system to achieve a 120 degrees/sec roll rate. The deformations of each of the wings required to achieve the specified roll rate are shown in Fig. 5 and Fig. 6.

This control system was designed for several Mach numbers and the corresponding dynamic pressure for roll maneuvers at 20,000 ft. The strain

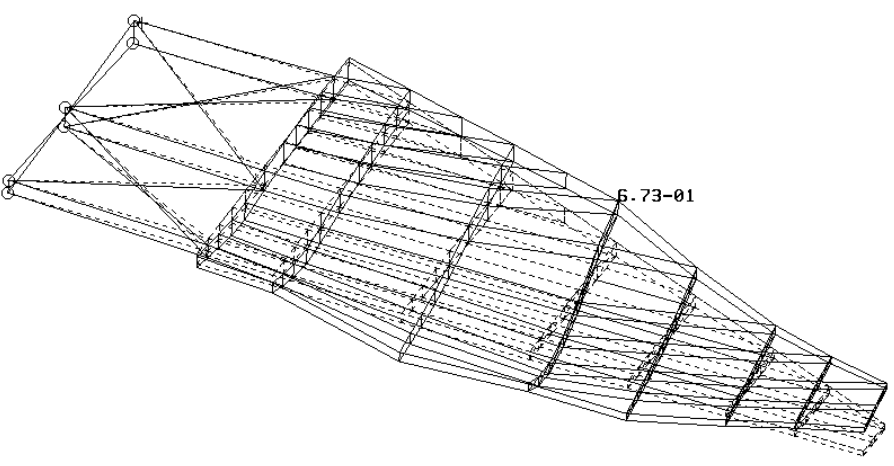

Figure 5. Deformation of Wing 1 needed to achieve roll rate of 120 degrees/sec at Mach 2.0 energy required to warp the wing is also computed and is given in Table 2.

For both wings, as the dynamic pressure increases, the amount of energy required to warp the wing decreases. This is as expected and demonstrates how the flexibility of the wing can be used to an advantage for maneuverability.

Since the first ten frequencies of the wings are similar (a constraint was placed on the first frequency to be greater than or equal to 3.0 ), it is concluded that the stiffnesses are also similar. The energy required to flex the higher aspect ratio wing (wing 2) is significantly less at the lower dynamic pressures, and at high dynamic pressures, the lower aspect

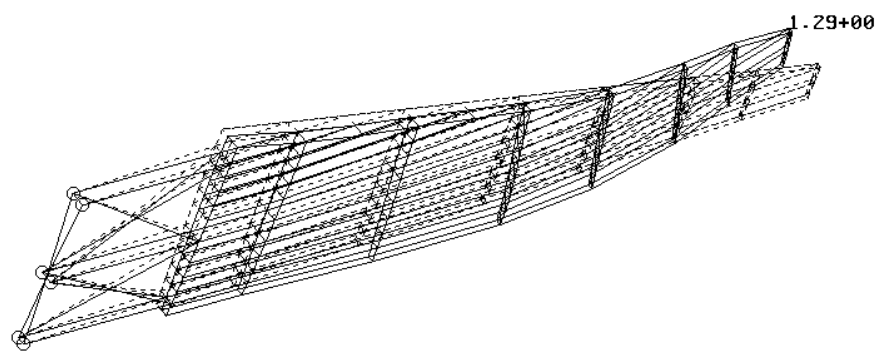

Figure 6. Deformation of Wing 2 needed to achieve roll rate of 120 degrees/sec at Mach 2.0 ratio wing requires less energy to achieve the specified roll rate.

It is believed that this occurs because of the difference in roll damping between the two wings. Roll damping is the effect of the apparent angle of attack that the wing sees due to the rolling motion. For the same roll rate, flight condition and wing area, a higher aspect ratio wing has a higher roll damping. The beneficial effects of the flexibility of the high aspect ratio wing are competing against its higher roll damping. At lower speeds, the flexibility benefits outweigh the roll damping.

\section{Summary}

The linking of data between various levels of analysis using AML (e.g., linking conceptual design parameters to the preliminary design models) allows data decks of more advanced analysis packages to be generated more quickly thus allowing quicker turn around on the higher level analyses. If the time to modify existing models is significantly reduced, the effects of conceptual level decisions on more detailed designs will be significantly better understood.

While this effort demonstrates the potential for quick turn around of finite element and aerodynamic models for changes in planform geometry, the AML-ASTROS GUI still requires much work to enhance the robustness of the program and the flexibility of the program. For example, the program is currently limited in the planform and airfoil definitions available. The definition of the extra points and boundary conditions could be modified to enhance the dependent relationships so that they do not need to be redefined when the planform geometry changes.

This paper shows the benefit of being able to quickly incorporate a planform change into the finite element model. The optimum planform and substructure design for roll control with this concept are linked. The substructure design effects the stiffness and the planform effects the roll damping. The design process to exploit this concept must allow for planform and substructural design simultaneously. 
Table 2: Strain Energy Requirements to Achieve $120 \mathrm{deg} / \mathrm{sec}$ Flexible Roll Rate (Altitude $20,000 \mathrm{ft})$

\begin{tabular}{|c|c|c|c|}
\hline Mach & $\begin{array}{c}\text { Pressure } \\
\left(\mathrm{lb}_{\mathrm{in}}{ }^{2}\right)\end{array}$ & $\begin{array}{c}\text { Strain Energy } \\
(\mathrm{ft} \mathrm{lbs})\end{array}$ & $\begin{array}{c}\text { Strain Energy } \\
(\mathrm{ft} \text { lbs })\end{array}$ \\
\hline \hline 1.20 & 6.81 & 2427 & 1537 \\
\hline 1.40 & 9.27 & 1945 & 985 \\
\hline 1.60 & 12.1 & 1519 & 862 \\
\hline 1.80 & 15.3 & 1186 & 775 \\
\hline 2.00 & 18.9 & 935 & 657 \\
\hline 2.20 & 22.9 & 749 & 580 \\
\hline 2.40 & 27.2 & 606 & 560 \\
\hline 2.60 & 32.0 & 499 & 550 \\
\hline 2.80 & 37.1 & 417 & \\
\hline 3.00 & 42.6 & 353 & \\
\hline
\end{tabular}

a. Wing 1

b. Wing 2

\section{References}

1. Veley, D. E., Blair, M., and Zweber, J. V., “Aerospace Technology Assessment System,” AIAA Paper 98-4825, Proceedings of the 7th AIAA/USAF/NASA/ISSMO Symposium on Multidisciplinary Analysis and Optimization, St. Louis, MO, September 2-4, 1998.

2. Blair, M., "Enabling Conceptual Design in a Technology-Driven Environment," AIAA Paper 98-4741, Proceedings of the 7th AIAA/USAF/NASA/ISSMO Symposium on Multidisciplinary Analysis and Optimization, St. Louis, MO, Sept. 2-4, 1998.

3. Zweber, J. V., and Hartong, A. R., "Structural and Control Surface Design for Wings Using the Adaptive Modeling Language," AIAA Paper 98-4869, Presented at the 7th AIAA/USAF/NASA/ISSMO Symposium on Multidisciplinary Analysis and Optimization, St. Louis, MO, September 2-4, 1998.

4. ASTROS User's Reference Manuel for Version 20, Universal Analytics, Inc., Torrance, CA, 1997.

5. Veley, D. E. and Zweber, J. V., “Automatic Differentiation in the Adaptive Modeling Language," Proceedings of the Australasian Conference on Structural Optimization, Sydney, Australia, February 11-13, 1998.

6. Veley, D. E., "Optimization in the Adaptive Modeling Language," AIAA Paper 98-4872, Proceedings of the 7th AIAA/USAF/NASA/ISSMO Symposium on Multidisciplinary Analysis and Optimization, St. Louis, MO, September 2-4, 1998.

7. Khot, N. S., Eastep, F., and Kolonay, R. M., "A Method for Enhancement of the Rolling Maneuver of a Flexible Wing," AIAA Paper 96-1391, Proceedings of the 37th Structures, Structural Dynamics and Materials Conference, Salt Lake City, UT, April, 1996.

8. Woodward, F. A., "An Improved Method for the Aerodynamic Analysis of Wing-Body-Tail Configurations in Subsonic and Supersonic Flow,” NASA CR-2228, Part I, Theory and Application, May 1973. 